\title{
Hypophosphataemia in Septicaemia: Higher Incidence in Gram-negative than in Gram-positive Infections
}

\author{
G. F. RIEDLER,* M.D. ; W. A. SCHEITLIN,* M.D.
}

\begin{abstract}
Ummary : Phosphataemia has been investigated in 54 patients with Gram-negative septicaemias ; either absolute (serum phosphate $<2 \mathrm{mg}$. $/ 100 \mathrm{ml}$.) or relative (P./B.U.N. 0.04) hypophosphataemia was found in $69 \%$ of all determinations. In 37 patients with Gram-positive septicaemias hypophosphataemia was present in only $24 \%$, many of whom had additional Gram-negative bacteriuria and possible Gram-negative septicaemia suppressed by antibiotics. The mechanism of this phenomenon is not known. This new sign of Gram-negative septicaemia has some diagnostic importance.
\end{abstract}

\section{Introduction}

Though numerous non-specific features have been described in Gram-negative septicaemia, only the demonstration of pathogenic organisms in the bloc! is conclusive evidence of its presence. Signs of acute infection, acute circulatory failure with shock, or acute renal failure may be the major presenting syndromes. Recent reports have suggested that this severe and often fatal syndrome occurs in an increasing incidence (Weil et al., 1964).

We have previously described the frequent occurrence of marked hypophosphataemia in the acute stage of Gram-negative septicaemia (Scheitlin and Frick, 1964). The following study of a larger group of patients deals with the incidence of this phosphataemia in septicaemia caused by both Gram-negative and Gram-positive pathogenic organisms.

\section{Patients Studied and Methods Used}

The analysis concerns those patients admitted to the department of medicine of the University Hospital, Zurich, from 1960 to 1967 with acute septicaemia and positive blood cultures. Out of a total of 141 cases of septicaemia only the 91 who had simultaneous determinations of blood urea nitrogen and inorganic phosphate are included in this partly retrospective study.

In most cases urea was determined in the autoanalyser and the blood urea nitrogen value was calculated. In some patients with non-protein nitrogen determinations the blood urea nitrogen was calculated by the formula of Van Slyke (Peters and Van Slyke, 1946). Blood glucose was determined by a glucose-oxidase method. The serum inorganic phosphates were determined colorimetrically with an autoanalyser. Normal ranges for blood urea nitrogen are $9.5-20 \mathrm{mg} . / 100 \mathrm{ml}$., for inorganic phosphate $2.5-3.5 \mathrm{mg} . / 100 \mathrm{ml}$., and for glucose 80-105 mg./100 ml.

The patients studied included 50 with Gram-negative septicaemia, three on more than one occasion; 41 patients were more than 50 years old. The source of infection was located in the urogenital system in four-fifths of the cases. Gram-positive septicaemia was studied in 37 episodes occurring in 36 patients (Table I) ; 24 patients were more than 50 years old. The sources of infection were pyogenic foci in $30 \%$ and urinary tract infections in only $20 \%$.

* Consultant in Nephrology, Kantonsspital, Zurich.

\section{Results}

Absolute hypophosphataemia is arbitrarily defined as a phosphate value of $2 \mathrm{mg}$. $/ 100 \mathrm{ml}$. or less. In patients with renal failure and blood urea nitrogen of more than $50 \mathrm{mg} . / 100 \mathrm{ml}$. the ratio phosphate/urea nitrogen (P./B.U.N.) was evaluated irrespective of the presence of absolute hypophosphataemia, a decrease of this ratio below 0.04 being designated as relative hypophosphataemia. This index was $0.06 \pm 0.01$ in 70 determinations during acute, non-traumatic, and chronic renal failures without septicaemia, this being in accordance with the findings of Doolan et al. (1960).

TABLE I.-Hypophosphataemia in Gram-negative and Gram-positive Septicaemia

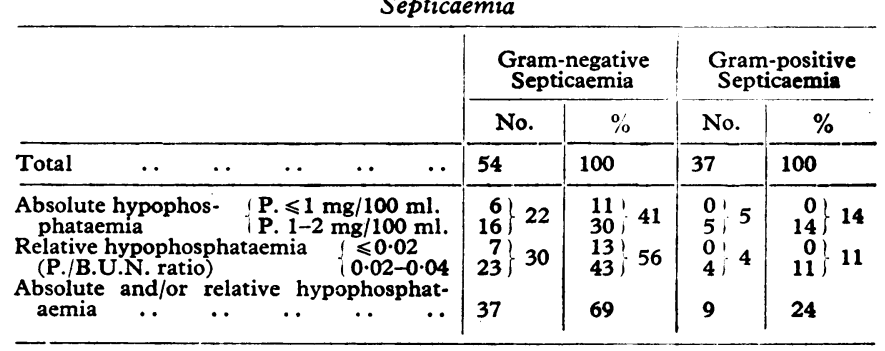

\section{Gram-negative Septicaemia}

General Findings.-Hypotension with systolic blood pressure values below $90 \mathrm{~mm}$. $\mathrm{Hg}$ was present in 38 of the $54 \mathrm{Gram}$ negative septicaemias, and in 29 cases this was followed by acute renal failure with oliguria or anuria (Table II). Twenty patients died, the main cause of death being toxic circulatory failure ( $90 \%$ of cases of death). Escherichia coli was found to be the pathogenic organism in $52 \%$ of all Gram-negative septicaemias. Proteus, pseudomonas, and klebsiella were found in about equal numbers; some rare pathogenic organisms and mixed infections were also found.

Hypophosphataemia.-Absolute hypophosphataemia was present in $41 \%$ of Gram-negative septicaemias (Table I), and in a few cases this marked hypophosphataemia could be shown even in the presence of severe azotaemia with blood urea nitrogen values of up to $125 \mathrm{mg} . / 100 \mathrm{ml}$. A relative hypophosphataemia with a P./B.U.N. ratio of less than 0.04 was observed in $56 \%$ of all Gram-negative septicaemias; $69 \%$ showed absolute or relative hypophosphataemia, or both (Fig. 1, Table I). In some cases the blood phosphorus concentration decreased within a few hours after the onset of septicaemia (Fig. 2). Phosphataemia became normal within days as the symptoms of acute septicaemia subsided (Figs. 2 and 3 ). Of the patients with absolute or relative hypophos-

TABLE II.-Relation of Clinical Course of Septicaemia to Phosphataemia

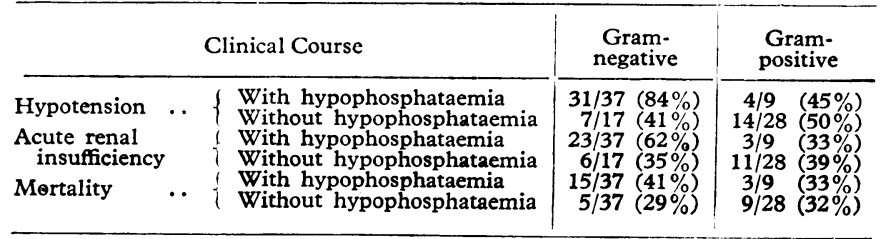


phataemia, $84 \%$ were hypotensive at the time of blood sampling or shortly before, while only $41 \%$ of those without low phosphate values showed hypotension (Table II). The mortality rate was $29 \%$ for patients without and $41 \%$ for patients with hypophosphataemia. In 12 out of 17

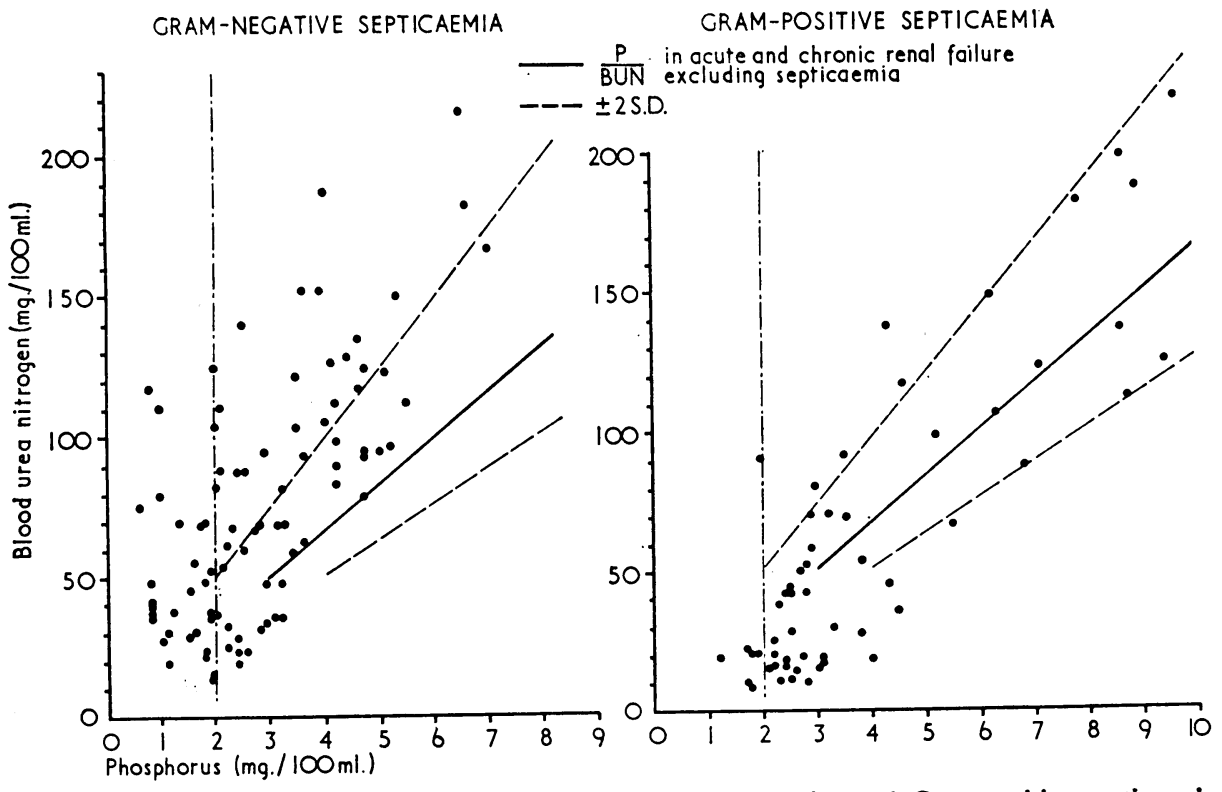

FIG. 1.-Phosphorus to blood urea nitrogen ratio in Gram-negative and Gram-positive septicaemia. Bach symbol represents the simultaneous determination of phosphorus and B.U.N. during the acute Bach symbol represents the simul line shows the regression line. The broken lines show the two stage of septicaemia. The solid line shows the regression line. The broken lines sho standard deviations in acute or chronic renal failure without septicaemia.
stand

\section{Gram-positive Septicaemias}

General Findings. - Transient hypotension with systolic blood pressure values below $90 \mathrm{~mm}$. $\mathrm{Hg}$ could be demonstrated in 18 of the 37 Gram-positive cases ; 14 patients suffered from acute renal failure with oliguria or anuria (Table II). Twelve patients died, the cause of death being toxic circulatory failure in $80 \%$. Pathogenic organisms such as Staphylococcus aureus were found in 17 patients, streptococci in 11 patients; the remainder of the cases showed pneumococci, enterococci, as well as sporadic mixed infections.

Hypophosphataemia. - Absolute hypophosphataemia with phosphate values up to $2 \mathrm{mg} . / 100 \mathrm{ml}$. was present in $14 \%$, while relative hypophosphataemia with P./B.U.N. ratio up to 0.04 was present in $11 \%$ of cases (Table I). Absolute or relative hypophosphataemia was found in $24 \%$ of Gram-positive

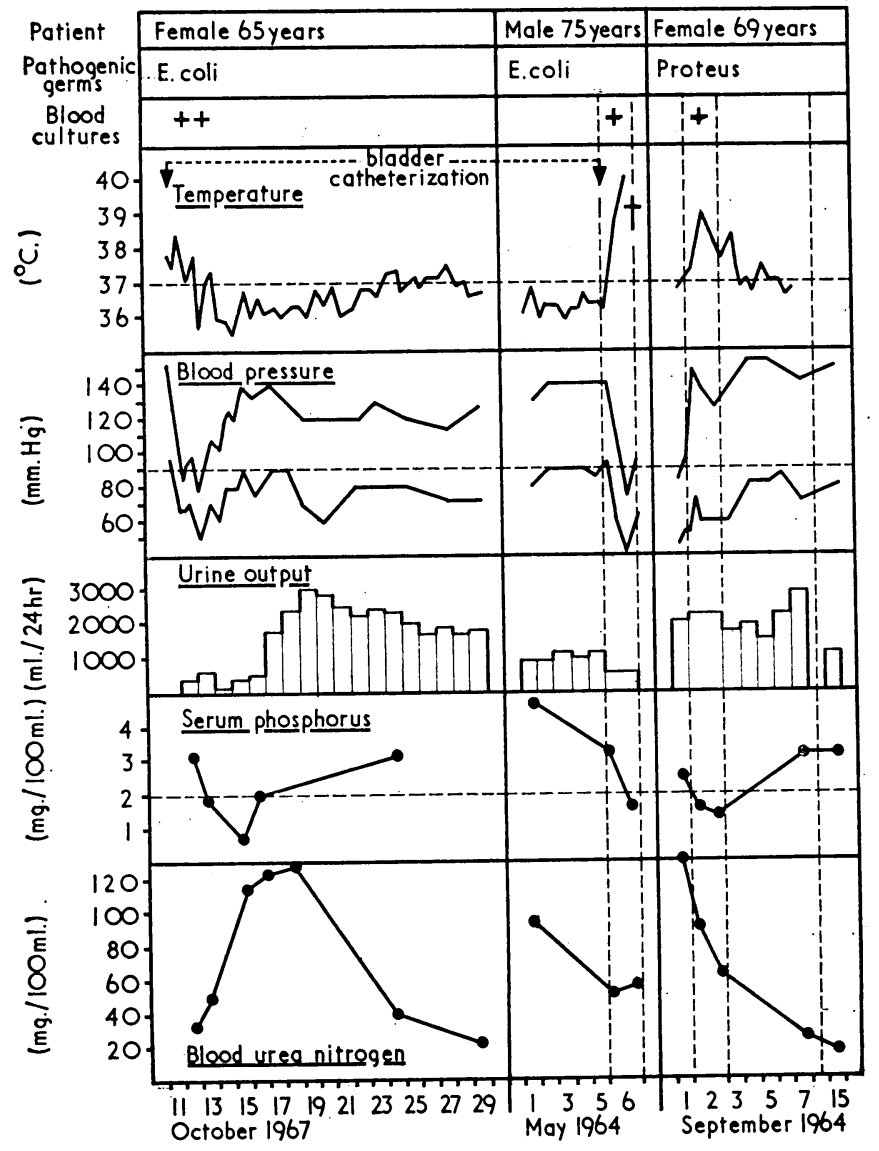

FIG. 2

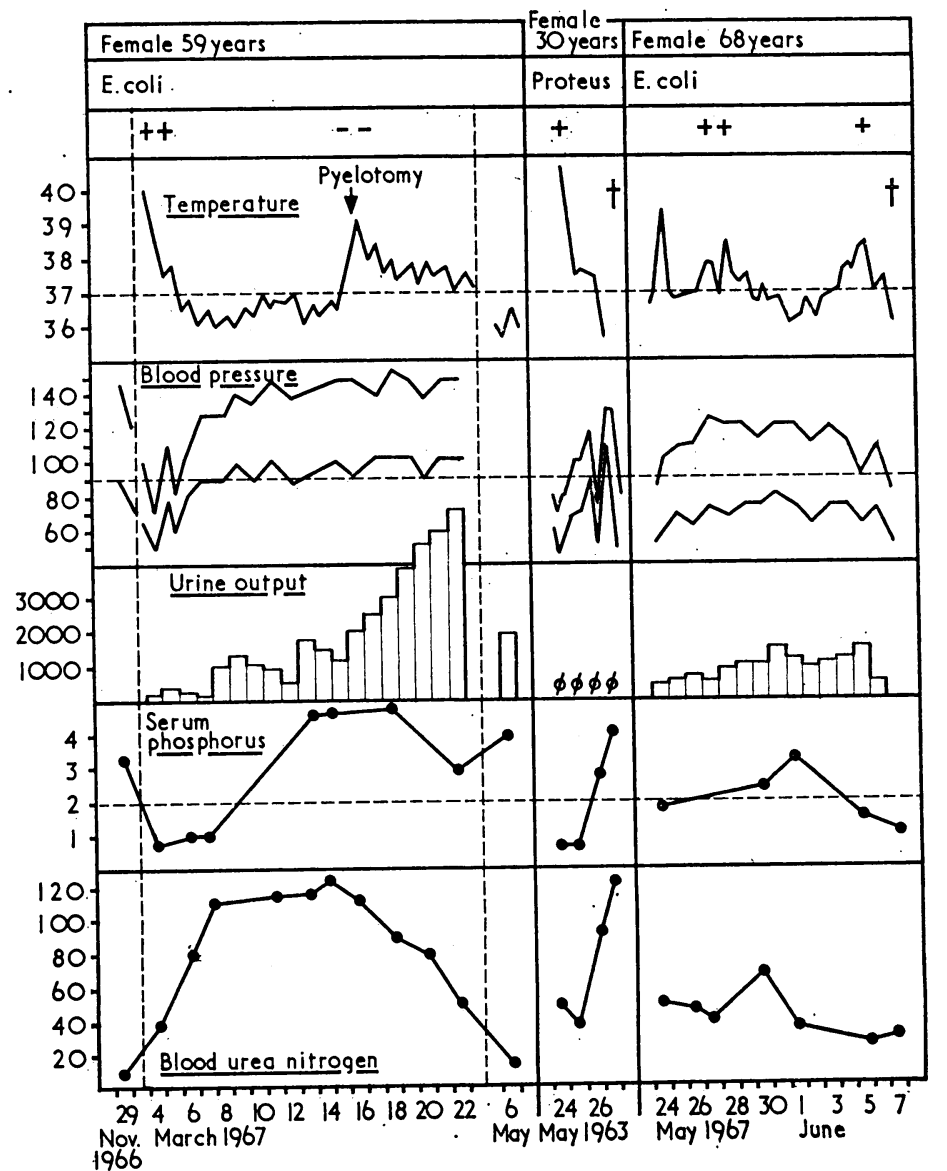

FIG. 3

FIG. 2-Clinical course and serial phosphorus determinations in three patients with acute Gram-negative septicaemia. Rapid occurrence of hypoFIG. 2.- Clinical course and serial phosphorus determinations in three patients with phosphorus determinations in three patients with acute Gramphosphataemia after the onset of septicaemia. FIG. 3.- Clinical course and serial phosphorus determinations in three patients with actiate or anuria and azotaemia. negative septicaemia. Occurrence of marked hypophosphataemia in the presence of acute renal failure with oliguria or
Recurrence of septicaemia in the third patient shows evidence of relapsing hypophosphataemia. 
septicaemias ; in six of the nine patients with absolute or relative hypophosphataemia there was also a simultaneous significant bacteriuria with Gram-negative organisms, yet the blood culture showed only Gram-positive pathogens. It is assumed that when there was a mixed infection with both kinds of organisms the Gram-positive organisms overgrew the Gram-negative or the latter were suppressed by antibiotic therapy; then only 3 out of 31 patients with pure Gram-positive septicaemia are left with hypophosphataemia. Of the nine patients with hypophosphataemia and the 28 patients with normal phosphate values, 4 and 14 respectively showed a reduction of systolic blood pressure to below $90 \mathrm{~mm}$. $\mathrm{Hg}$ (Table II). Mortality in the normal and low phosphate group was similar (Table II). Simultaneous determination of inorganic phosphate and calcium in the serum showed that four out of five patients had hypocalcaemia together with hypophosphataemia.

\section{Other Chemical Analyses}

Arterial blood gas analyses were performed simultaneously with the phosphate determinations in a group of eight patients with hypophosphataemia (seven with Gram-negative septicaemia) and in a group of 10 patients without reduced phosphate values (nine with Gram-positive septicaemia). Both groups showed a metabolic acidosis with similar findings. The mean $\mathrm{pH}$ value was $7 \cdot 32 \pm 0 \cdot 12$ in the cases with hypophosphataemia and $7 \cdot 32 \pm 0.13$ in the cases with normal phosphate values respectively; $\mathrm{CO}_{2}$ tension was $29.4 \pm 12.5 \mathrm{~mm}$. $\mathrm{Hg}$ and $29.6 \pm 7.0 \mathrm{~mm}$. $\mathrm{Hg}$ respectively; and the standard bicarbonate was $17 \cdot 7 \pm 5.9 \mathrm{mEq} / 1$. and $17 \cdot 8 \pm 4.8 \mathrm{mEq} / 1$. respectively. The average blood sugar value was $176 \pm 23 \mathrm{mg} . / 100 \mathrm{ml}$. in 23 patients without reduced phosphates and $178 \pm 56 \mathrm{mg} . / 100 \mathrm{ml}$. in 28 patients with reduced phosphates. Comparison of liver function studies with phosphate determinations showed similar values in both groups. The mean value for bilirubin in 28 patients with hypophosphataemia was $2 \cdot 0 \pm 2.0 \mathrm{mg} . / 100 \mathrm{ml}$. and $1.4 \pm 1.8 \mathrm{mg} . / 100 \mathrm{ml}$. in 33 patients without decreased phosphate; serum aspartate aminotransferase was $36 \pm 39$ i.u. (19 patients) and $39 \pm 21$ i.u. (20 patients) respectively.

\section{Discussion}

In 54 patients with Gram-negative septicaemia absolute $(41 \%)$ or relative $(56 \%)$ hypophosphataemia, or both, was present in $69 \%$. Only $24 \%$ of patients with Gram-positive septicaemia showed similar hypophosphataemia-a significant difference $(P<0.01)$. The cause of these findings is not clear. Acute renal loss of phosphate can be excluded, as $62 \%$ of our cases with hypophosphataemia presented acute renal failure with oliguria or anuria. Some patients developed severe hypophosphataemia within a few hours. The occurrence of extreme hypophosphataemia below $0.7 \mathrm{mg} . / 100 \mathrm{ml}$. with simultaneous acute renal failure and azotaemia must be stressed since hyperphosphataemia would normally be expected. Marked hypophosphataemia is described in hyperventilation and respiratory alkalosis (Robin et al., 1959 ; Saltzman et al., 1963 ; George et al., 1964 ; Mostellar and Tuttle, 1964). Some of our patients did show hyperventilation with $\mathrm{PCO}_{2}$ values as low as $15 \mathrm{~mm}$. $\mathrm{Hg}$, yet associated with metabolic acidosis. Thus the findings in respiratory alkalosis cannot be compared with the situation in Gram-negative septicaemia. Furthermore, we have observed severe hypophosphataemia in the presence of normal blood gases. A slight decrease of serum calcium corresponded to the azotaemia and the metabolic acidosis, yet 12 out of 17 patients showed simultaneously absolute hypophosphataemia and hypocalcaemia. A clear correlation between these two findings could not be established.

The observations of Froesch et al. (1963) in hereditary fructose intolerance appear to be important. In this metabolic disorder oral and intravenous fructose administration leads to a marked hypophosphataemia of 0.9 to $1.4 \mathrm{mg} . / 100 \mathrm{ml}$. within an hour. The authors believed that this was due to the deficiency in the fructose-1-phosphate-splitting enzyme aldolase and to an accumulation of the phosphate. A similar mechanism causing hypophosphataemia in Gram-negative septicaemia is possible.

Intravenous administration of glucose in metabolically healthy individuals can also lead to a decrease of inorganic phosphate (Cohen, 1962). The intravenous infusions given to our patients, however, do not explain the hypophosphataemia, since the latter could often be demonstrated before this therapy was started ; in our patients with Gram-negative septicaemia, hypophosphataemia was found in 22 out of 29 cases before any treatment was given and in 15 out of 25 cases after infusion was started. In patients with Gram-positive septicaemia decreased phosphorus levels were present in 4 out of 17 patients before and in 5 out of 25 patients after the beginning of infusion. The significant difference in behaviour of the serum phosphate levels in Gram-negative and Gram-positive bacteraemia, in spite of the same infusion policy for both groups, excludes this explanation as well.

A moderate increase of blood sugar was observed in our patients, irrespective of variety of organisms and concentration of phosphate. We consider a disturbance of the carbohydrate metabolism caused by Gram-negative endotoxin and the resulting blocking of phosphorus metabolism-analogous to the circumstances found in fructose intolerance-is a possible explanation of hypophosphataemia. This theory, however, requires further investigation. Disturbances of liver function can often be shown in Gram-negative septicaemia (Weil et al., 1964 ; Kenel et al., 1967). In our cases of Gram-negative septicaemia and hypophosphataemia, serum aspartate aminotransferase was slightly increased. At the same time a mild hyperbilirubinaemia was found. The interpretation of the liver tests is, however, difficult in this severe and complex clinical syndrome, which is often associated with haemolysis (Rapaport et al., 1964), defibrination (Rapaport et al., 1964 ; Rosner and Ritz, 1966 ; McGehee et al., 1967 ; Tönz et al., 1967), hypotension (Oechslin et al., 1962 ; Spink, 1962 ; Weil et al., 1956, 1964 ; Waisbren, 1964 ; Hodgin and Sanford, 1965 ; Anderson et al., 1967), and renal insufficiency (Weil et al., 1964 ; Strauch et al., 1967).

The observation of these apparently specific blood-chemical aberrations of severe absolute or relative hypophosphataemia in Gram-negative septicaemia is of some diagnostic significance. In numerous patients, not included in this study, hypophosphataemia corresponded to the clinical and post-mortem findings of Gram-negative septicaemia without demonstration of bacteraemia. The demonstration of pathogenic organisms in peripheral blood is often impossible because of the previous administration of antibiotics. Thus the finding of hypophosphataemia in suspected Gram-negative septicaemia adds weight to this suspicion. Early and effective therapy can reduce the mortality of this dangerous clinical syndrome (Weil et al., 1964 ; Hodgin and Sanford, 1965). Hypophosphataemia with fever following chills or with hypotension or urinary tract infection is almost proof of Gram-negative septicaemia in our experience.

The recognition of a disturbed phosphorus metabolism in Gram-negative septicaemia may also be of therapeutic value. The investigations of Garner et al. (1967) showed that the mortality of guinea-pigs infected with salmonellae will be reduced by the administration of phosphate. As a matter of fact, these authors described a decrease of blood phosphates before death of the animals. When phosphate was supplied, mortality was significantly reduced and the number of organisms found in the tissue decreased. We suggest investigations on the therapeutic value of phosphate infusions in patients with Gram-negative septicaemia.

Requests for reprints should be sent to Dr. W. A. Scheitlin. 
REFERENCES

Anderson, R. W., James, P. M., Bredenberg, C. E., and Hardaway, R. M. (1967). Ann. Surg., 165, 341.

Cohen, B. D. (1962). Ann. intern. Med., 57, 204.

Doolan, P. D., Wiggins, R. A., Thiel, G. B., Lee, K. J., and Martinez, E. (1960). Amer. f. Med., 28, 895 . Froesch E. R., Wolf, H. P., Baitsch, H., Prader, A., and Labhart, A.

Garner, G. B., Huebner, P. F., and O'Dell, B. L. (1967). Fed. Proc., 26, 799.

George, W. K., George, W. D., Smith, J. P., Gordon, F. T., Baird, Hodgin U. G and Sanfond J P. (1965). Amer. 7. Med. 39, 952.

Hodgin, U. G., and Sanford, J. P. (1965). Amer. f. Med., 39, 952

Kenel, F., et al. (1967). Schweiz. med. Wschr., 97, 877.

McGehee, W. G., Rapaport, S. I., and Hjort, P. F. (1967). Ann. intern. Med., 67, 250 .

Mostellar, M. E., and Tuttle, E. P. (1964). F. clin. Invest., 43, 138.
Oechslin, R., Scheitlin, W., and Frick, P. (1962). Schweiz. med. Wschr., 92, 1151 .

Peters, J. P., and Van Slyke, D. D. (1946). Quantitative Clinical Chemistry Interpretations. Williams and Wilkins, Baltimore.

Rapapont, S. I., Tatter, D., Coeur-Barron, N., and Hjont, P. F. (1964). New Engl. 7. Med., 271, 80.

Robin, E. D., Davis, R. P., and Rees, S. B. (1959). Amer. F. Med., 26, 869.

Rosner, F., and Ritz, N. D. (1966). Arch. intern. Med., 117, 17.

Saltzman, H. A., Heyman, A., and Sieker, H. O. (1963). New Engl. f. Med., 268, 1431 .

Scheitlin, W. A., and Frick, P. G. (1964). Lancet, 2, 102.

Spink, W. W. (1962). Ann. intern. Med., 57, 538.

Strauch, M., et al. (1967). Ann. Surg., 165, 536.

Waisbren, B. A. (1964). Amer. F. Med., 36, 819.

Weil, M. H., MacLean, L. D., Visscher, M. B., and Spink, W. W. (1956). f. clin. Invest., 35, 1191 .

Weil, M. H., Shubin, H., and Biddle, M. (1964). Ann. intern. Med., 60, 384.

\title{
Ileorectal Anastomosis: Appreciation by Patients
}

\author{
D. G. JAGELMAN, $*$ M.B., B.S. ; C. B. LEWIS, † F.F.A. R.C.S., D.A. ; D. C. ROWE-JONES, $\ddagger$ M.B., F.R.C.S.
}

Brit. med. F., 1969, 1, 756-757

\begin{abstract}
Cummary : Two hundred patients treated by ileorectal $D$ anastomosis for ulcerative colitis were questioned about their opinion of the result of their operation. The vast majority of patients led normal business and social lives and their activities had been greatly altered for the better by this operation.
\end{abstract}

\section{Introduction}

The treatment of ulcerative colitis remains a challenge to physician and surgeon alike. Ideally cases should be managed by a medical/surgical team, since this provides experience from both disciplines and helps to prevent the therapeutic problem being conditioned by individual prejudice. In spite of extensive research the aetiology remains unknown, and in consequence our chief advances in the understanding of the disorder have been in the fields of supportive therapy and prognosis. Ultimately, however, many cases reach a point of no return, when it becomes clinically obvious that removal of the colon must be undertaken in order to save the patient's life.

When the decision to operate has been made, two main lines of surgical treatment exist-namely, the removal of the whole colon and rectum (panproctocolectomy) with the formation of an ileostomy or colectomy and ileorectal anastomosis with preservation of the anus. The merits and demerits of the two main lines of surgery are well recognized, and much contentious argument has resulted from discussion of these. The panproctocolectomy by its very nature precludes any further disease of the colon and rectum, but the patient is left with a permanent ileostomy. Ileorectal anastomosis on the other hand has the great advantage of leaving the patient with an anus, but the procedure leaves the patient open to the risk of continuing or subsequent disease in the rectal stump. In this paper it is not proposed to reiterate the technical and prognostic points made by both schools of thought, but merely to report the opinions of a large group of

- Surgical Registrar, Westminster Hospital, London S.W.1.

† Consultant Anaesthetist, Westminster Hospital ; Senior Anaesthetist, Royal Marsden Hospital, London S.W.3.

‡ Senior Surgical Registrar, Westminster Hospital, London S.W.1. ulcerative colitic patients who had undergone the operation of ileorectal anastomosis.

\section{Method}

Contact with the patients was made by postal questionary with an accompanying brief letter requesting them to return the completed forms in a stamped addressed envelope provided. The letter was signed by a physician colleague, who was completely unknown to any of the recipients. No clue was given concerning the purpose of the investigation other than that the information was required for research purposes of a surgical nature, and not of ileorectal anastomosis in particular. Patients were selected at random from a large series of whom about $50 \%$ were incorporated into the inquiry. The only stipulation made was that they must have undergone a period of at least 12 months since the completion of one or more surgical procedures which culminated in their being discharged with a functioning ileorectal anastomosis. We are fully conscious of the defects introduced by any form of questionary, depending as it does on the intelligence of the patient and the difficulty of framing questions that mean the same thing to a wide variety of people. However, it was felt important to maintain the inquiry at as impersonal a level as possible, and, furthermore, by this method of communication it was hoped to diminish any element of gratitude or hospital/ surgeon attachment.

\section{The Patients}

Two hundred patients were selected at random and a questionary was sent to each of them as described. All cases would be classified as seriously ill at the time of operation and, in particular, had failed to respond to all forms of medical treatment available at the appropriate time. They came from all over the British Isles. A fortnight was allowed for the patient to respond to the initial inquiry, and, failing an answer within that time, a further questionary was sent with a reminding request. No further action was taken. The questionary was designed with relative simplicity as the keynote. The three preoperative questions were formulated to evaluate the patient's physical, mental, and economic state during this 